\title{
An Efficient Numerical Algorithm for Solving Fractional Higher-Order Nonlinear Integrodifferential Equations
}

\author{
Muhammed I. Syam, Qasem M. Al-Mdallal, and M. Naim Anwar \\ Department of Mathematical Sciences, United Arab Emirates University, P.O. Box 15551, Al-Ain, UAE \\ Correspondence should be addressed to Muhammed I. Syam; m.syam@uaeu.ac.ae
}

Received 3 July 2015; Accepted 11 October 2015

Academic Editor: Jozef Banas

Copyright ( 2015 Muhammed I. Syam et al. This is an open access article distributed under the Creative Commons Attribution License, which permits unrestricted use, distribution, and reproduction in any medium, provided the original work is properly cited.

This paper is devoted to both theoretical and numerical study of boundary value problems for higher-order nonlinear fractional integrodifferential equations. Existence and uniqueness results for the considered problem are provided and proved. The numerical method of solution for the problem is based on a conjugate collocation and spline approach combined with shooting method. Some numerical examples are discussed to demonstrate the efficiency and the accuracy of the proposed algorithm.

\section{Introduction}

Within the context of fractional calculus, it is argued that anticipated sort of memory is being carried out from past states to current states; see, for example, the recent work of Agarwal et al. [1]. Therefore, in recent years, several phenomena in physics, chemistry, life sciences, geophysics and earth sciences, and fluid dynamics have been extensively investigated through mathematical models involving fractional calculus; see, for example, the works by Podlubny [2], Mainardi [3], and Kilbas et al. [4].

In this paper we consider a class of boundary value problems for nonlinear integrodifferential equations of the form

$$
\begin{aligned}
\mathscr{L} y:=D^{\alpha} y(x)+\int_{0}^{x} K(x, t) f(t, y) d t+ & h(x)=0, \\
x \in I & =[0,1],
\end{aligned}
$$

subject to

$$
\begin{gathered}
y(0)=a_{0}, \\
y^{\prime}(0)=a_{1} \\
y(1)=b_{0}, \\
y^{\prime}(1)=b_{1},
\end{gathered}
$$

where $3<\alpha \leq 4, f \in C[I \times \mathbb{R}, \mathbb{R}], K \in C\left[I \times I, \mathbb{R}^{+}\right]$is a positive kernel, $h(x) \in C[I, \mathbb{R}]$, and $a_{1}, b_{0}, b_{1} \in \mathbb{R}$. Here, $D^{\alpha}$ denotes the fractional differential operator of order $\alpha$ in Caputo's sense and is given by

$$
D^{\alpha} y(x)=\frac{1}{\Gamma(k-\alpha)} \int_{0}^{x}(x-t)^{k-\alpha-1} y^{(k)}(t) d t,
$$

where $k=\lceil\alpha\rceil$ is the smallest integer greater than or equal to $k$. It is well-known that many mathematical models in engineering and other disciplines in science involve integrodifferential equations of fractional order, for example, problems in modeling of turbulent aerodynamic phenomena, continuum under viscoelastic situations, certain population dynamics problems, and heat transfer in composite materials with certain properties. Account for similar issues is given in [5-8] and the references therein.

A survey of the literature reveals that theoretical and numerical investigations related to corresponding boundary value problems for fractional differential and integrodifferential equations are still in their early stages; see [9-17] and the references therein. Moreover, it is extremely difficult to find exact solutions of such problems; therefore, most researchers have focused on the numerical methods to approximate exact solutions. Examples of such methods are the Adomian decomposition method [18, 19], collocation spline method [20] and Zhao et al. [21], variational iteration method and 
homotopy perturbation method $[22,23]$, fractional differential transform method [24, 25], CAS wavelets [26], discrete Galerkin method [27], Chebyshev wavelets method adopted by Biazar and Ebrahimi [28], and Taylor expansion method [29].

The rest of the paper is organized as follows: some definitions and preliminary results are presented in Section 2. In Section 3, some relevant theoretical results such as the existence and uniqueness of the considered problem are presented. The numerical method of solution is presented in Section 4. In Section 5, numerical examples are discussed to demonstrate the efficiency and the rapid convergence of the present algorithm.

\section{Definitions and Preliminary Results}

This section presents some definitions and preliminary results that will be extensively used in this study. We first introduce the Riemann-Liouville definition of fractional derivative operator.

Definition 1. The left sided Riemann-Liouville fractional integral operator of order $\alpha$ is defined by

$$
J^{(\alpha)} y(x)=\frac{1}{\Gamma(\alpha)} \int_{a}^{x}(x-t)^{\alpha-1} y(t) d t
$$

where $y \in L_{1}(a, b)$ and $\alpha \in \mathbb{R}^{+}$.

The properties of the operator $J^{(\alpha)}$ are summarized in the following lemma.

Lemma 2. Let $\alpha, \beta, x>0$ and $\gamma>-1$. Then, see [2]

(i) $\left(J^{(\alpha)} J^{(\beta)} y\right)(x)=\left(J^{(\alpha+\beta)} y\right)(x)=\left(J^{(\beta)} J^{(\alpha)} y\right)(x)$,

(ii) $J^{(\alpha)}(x-a)^{\gamma}=(\Gamma(\gamma+1) / \Gamma(\gamma+1+\alpha))(x-a)^{\gamma+\alpha}$.

Note that the left sided Caputo fractional derivative (3) is originally defined via the left sided Riemann-Liouville fractional integral (4), as follows:

$$
D^{(\alpha)} y(x)=J^{(n-\alpha)} y^{(n)}(x)=y(x), \quad x>0,
$$

where $\alpha \in \mathbb{R}^{+}, n=\lceil\alpha\rceil$, and $y \in L_{1}[a, b]$.

Lemma 3. For $\alpha \in \mathbb{R}^{+}, n=\lceil\alpha\rceil$, and $y \in L_{1}[a, b]$, one has

(i) $\left(D^{(\alpha)} J^{(\alpha)} y\right)(x)=y(x)$.

(ii) $\left(J^{(\alpha)} D^{(\alpha)} y\right)(x)=y(x)-\sum_{m=0}^{n-1} y^{(m)}\left(a^{+}\right)\left((x-a)^{m} / m !\right)$.

(iii) $D^{(\alpha)}(x-a)^{r}=(\Gamma(r+1) / \Gamma(r+1-\alpha))(x-a)^{r-\alpha}$, for $[\alpha]<r$.

\section{Analytical Results}

The existence and uniqueness of the exact solution to problem (1) subject to the boundary conditions (2a) and (2b) are discussed herein. Since we are using the shooting method which requires converting the boundary value problem to initial value problem, we will discuss in the next theorem the existence of the solution to (1) subject to

$$
\begin{gathered}
y(0)=a_{0}, \\
y^{\prime}(0)=a_{1}, \\
y^{\prime \prime}(0)=a_{2}, \\
y^{\prime \prime \prime}(0)=a_{3} .
\end{gathered}
$$

Theorem 4 (existence). Assume that $h \in C[0,1], k \in C[0,1]^{2}$, and $f \in C[0,1] \times \mathbb{R}$. Then for any $\epsilon>0$ and

$$
\chi=\min \left\{1,\left(\frac{\epsilon \Gamma(\alpha+1)}{\|h\|_{\infty}+\|K\|_{\infty}\|f\|_{\infty}}\right)^{1 / \alpha}\right\}
$$

there exists $y:[0, \chi] \rightarrow \mathbb{R}$ solving the initial value problem (1) and (6).

Proof. Taking the Riemann-Liouville functional operator of both sides of (1) and applying Lemma 3, one obtains

$$
\begin{aligned}
y(x)= & \sum_{i=0}^{3} \frac{y^{(i)}(0)}{i !} x^{i} \\
& -\frac{1}{\Gamma(\alpha)} \int_{0}^{x} \frac{h(\tau)+\int_{0}^{\tau} K(\tau, t) f(t, y) d t}{(x-\tau)^{1-\alpha}} d \tau .
\end{aligned}
$$

Let $\mathbf{B}=\left\{y \in C[0, \chi]:\left\|y-\sum_{i=0}^{3}\left(y^{(i)}(0) / i !\right)\right\|_{\infty}<\epsilon\right\}$. Obviously, $\mathbf{B}$ is a closed subset of the Banach space of all continuous functions on $[0, \chi]$ equipped with the Chebyshev norm. Moreover, since $y(x)=\sum_{i=0}^{3}\left(y^{(i)}(0) / i !\right) x^{i}$ for $x \in[0, \chi]$ is in $\mathbf{B}$ then $\mathbf{B} \neq \phi$. Define the operator $\mathscr{L}$ on $\mathbf{B}$ by

$$
\begin{aligned}
\mathscr{L}[y] & (x) \\
:= & \sum_{i=0}^{3} \frac{y^{(i)}(0)}{i !} x^{i} \\
& -\frac{1}{\Gamma(\alpha)} \int_{0}^{x} \frac{h(\tau)+\int_{0}^{\tau} K(\tau, t) f(t, y) d t}{(x-\tau)^{1-\alpha}} d \tau .
\end{aligned}
$$

The equation under consideration can be written as

$$
\mathscr{L}[y]=y .
$$

Our aim is to show that (10) has a fixed point in B. Since $h \epsilon$ $C[0,1], k \in C[0,1]^{2}$, and $f \in C[0,1] \times \mathbb{R}$, then $\mathscr{L}[y]$ is a continuous function. Notice that, since $2<\alpha \leq 4$ ensures that $\alpha-1>2$. To achieve our target, we need to show that $\mathscr{L}$ is a self-mapping on $\mathbf{B}$. For any $y \in \mathbf{B}$ and $x \in[0, \chi]$, we have

$$
\begin{aligned}
& \left|\mathscr{L}[y](x)-\sum_{i=0}^{3} \frac{y^{(i)}(0)}{i !} x^{i}\right| \\
& \quad=\frac{1}{\Gamma(\alpha)}\left|\int_{0}^{x} \frac{h(\tau)+\int_{0}^{\tau} K(\tau, t) f(t, y) d t}{(x-\tau)^{1-\alpha}} d \tau\right| \\
& \leq \frac{1}{\Gamma(\alpha)}\left|\int_{0}^{x} \frac{\|h\|_{\infty}+\|K\|_{\infty}\|f\|_{\infty} \tau}{(x-\tau)^{1-\alpha}} d \tau\right| .
\end{aligned}
$$


Since $0 \leq \tau \leq \chi \leq 1$, then

$$
\begin{aligned}
& \left|\mathscr{L}[y](x)-\sum_{i=0}^{3} \frac{y^{(i)}(0)}{i !} x^{i}\right| \leq \frac{1}{\Gamma(\alpha)}\left(\|h\|_{\infty}+\|K\|_{\infty}\right. \\
& \left.\cdot\|f\|_{\infty}\right)\left|\int_{0}^{x}(x-\tau)^{\alpha-1} d \tau\right|=\frac{1}{\Gamma(\alpha)}\left(\|h\|_{\infty}+\|K\|_{\infty}\right. \\
& \left.\cdot\|f\|_{\infty}\right) \frac{x^{\alpha}}{\alpha} \leq \frac{1}{\Gamma(\alpha+1)}\left(\|h\|_{\infty}+\|K\|_{\infty}\|f\|_{\infty}\right) \\
& \cdot \chi^{\alpha} \leq \frac{1}{\Gamma(\alpha+1)}\left(\|h\|_{\infty}+\|K\|_{\infty}\right. \\
& \left.\cdot\|f\|_{\infty}\right) \frac{\epsilon \Gamma(\alpha+1)}{\|h\|_{\infty}+\|K\|_{\infty}\|f\|_{\infty}} \\
& =\epsilon .
\end{aligned}
$$

Therefore, $\mathscr{L}[y] \in \mathbf{B}$ if $y \in \mathbf{B}$; that is, $\mathscr{L}$ maps $y$ to itself. Based on Banach's fixed point theorem, it follows that the proof is complete.

It is easy to see that the solution produced by Theorem 4 depends on $x, a_{2}$, and $a_{3}$. To prove the existence of solution to problem (1)-(2b), it is enough to force the solution to satisfy the condition (2b). In this case, we can determine values of $a_{2}$ and $a_{3}$ so that the solution of (1)-(2b) depends on $x$ only.

Theorem 5 (uniqueness). Let $f$ be a Lipschitz function in the variable $y$ with Lipschitz constant $L$. Let $K$ be a bounded function on $[0,1] \times[0,1]$ such that

$$
\left|K\left(t_{1}, t_{2}\right)\right| \leq M, \quad \forall\left(t_{1}, t_{2}\right) \in[0,1] \times[0,1],
$$

where $M>0$. If $0<L M / \alpha \Gamma(\alpha)<1$; then problem (1)-(2b) has a unique solution.

Proof. Let $y_{1}$ and $y_{2}$ be two solutions to problem (1)-(2b); then

$$
\begin{aligned}
& D^{\alpha} y_{1}(x)+\int_{0}^{x} K(x, t) f\left(t, y_{1}\right) d t+h(x)=0 \\
& D^{\alpha} y_{2}(x)+\int_{0}^{x} K(x, t) f\left(t, y_{2}\right) d t+h(x)=0 .
\end{aligned}
$$

Subtracting (14) from (15) then applying the RiemannLiouville fractional integration one obtains

$$
\begin{aligned}
& \left|y_{2}-y_{1}\right| \\
& \quad=\frac{1}{\Gamma(\alpha)}\left|\int_{0}^{x} \frac{\int_{0}^{\tau} K(\tau, t)\left(f\left(t, y_{2}\right)-f\left(t, y_{1}\right)\right) d t}{(x-\tau)^{1-\alpha}} d \tau\right| \\
& \quad \leq \frac{L M}{\Gamma(\alpha)}\left|y_{2}-y_{1}\right|\left|\int_{0}^{x} \frac{1}{(x-\tau)^{1-\alpha}} d t\right| \\
& \quad \leq \frac{L M}{\alpha \Gamma(\alpha)}\left|y_{2}-y_{1}\right| .
\end{aligned}
$$

Obviously,

$$
\left|\frac{1}{(x-\tau)^{1-\alpha}} d t\right|=\left|\frac{x^{\alpha}}{\alpha} \leq \frac{1}{\alpha}\right| .
$$

Since $L M / \alpha \Gamma(\alpha)<1$, then $y_{1}=y_{2}$ which completes the proof.

\section{Method of Solution}

The following is a brief derivation of the numerical algorithm used to solve problem (1) subject to (2a) and (2b). It is based on conjugate collocation approach with multiple shooting method. It consists of three main steps:
(1) Collocation step.
(2) Spline step.
(3) Multiple shooting step.

4.1. Collocation and Spline Methods. For the sake of simplicity, we discuss the solution of (1) as initial value problem with

$$
\begin{gathered}
y(0)=a_{0}, \\
y^{\prime}(0)=a_{1}, \\
y^{\prime \prime}(0)=a_{2}, \\
y^{\prime \prime \prime}(0)=a_{3},
\end{gathered}
$$

where $a_{2}$ and $a_{3}$ are unknown constants which will be determined later.

The interval $I=[0,1]$ is partitioned into $N$ uniform subintervals $\lambda_{i}=\left[x_{i-1}, x_{i}\right]$ (for $i=1,2, \ldots, N$ ) of width $h=$ $1 / N$. Let $Z_{N}=\left\{x_{i}=i h: i=1,2, \ldots, N-1\right\}$. For a given $m \geq$ 1 , let $S_{m+3}^{(3)}\left(Z_{N}\right)$ be the spline space of piecewise polynomials on $Z_{N}$ which are 3 times continuously differentiable on the interval $I$, given by

$$
\begin{aligned}
& S_{m+3}^{(3)}\left(Z_{N}\right) \\
& \quad=\left\{u \in C^{3}(I): u_{\left.\right|_{\lambda_{i}}}=u_{i}(t) \in \mathbb{P}_{m+3} \text { on } \lambda_{i}\right\},
\end{aligned}
$$

where $\mathbb{P}_{m+3}$ represents the set of all real polynomials of degree not exceeding $m+3$. Notice that $m$ represents the number of collocation points in each subinterval $\lambda_{i}$. Those points are defined as

$$
X_{i}=\left\{x_{i, j}=x_{i}+h c_{j}: i=0: N-1, j=1: m\right\},
$$

with $0 \leq c_{1} \leq c_{2} \leq \cdots \leq c_{m} \leq 1$. Based on the collocation method, the exact solution $y$ of problem (1) subject to (18) will be approximated by an element $u \in S_{m+3}^{(3)}\left(Z_{N}\right)$ such that

$$
\begin{aligned}
D^{\alpha} u(x)+\int_{0}^{x} K(x, t) f(t, u) d t+h(x) & =0, \\
x & \in X=\bigcup_{i=0}^{N-1} X_{i},
\end{aligned}
$$

subject to

$$
\begin{gathered}
u(0)=a_{0}, \\
u^{\prime}(0)=a_{1},
\end{gathered}
$$




$$
\begin{aligned}
& u^{\prime \prime}(0)=a_{2}, \\
& u^{\prime \prime \prime}(0)=a_{3} .
\end{aligned}
$$

On each subinterval $\lambda_{i}$, the spline $u$ can be expressed as a piecewise polynomial of degree $m+3$ of the form

$$
\begin{aligned}
& u(x)= u_{i}\left(x_{i}+h \chi\right) \\
&=\mu_{0}^{(i)}+\mu_{1}^{(i)} \chi+\mu_{2}^{(i)} \chi^{2}+\mu_{3}^{(i)} \chi^{3}+\sum_{j=1}^{m} \beta_{j}^{(i)} \chi^{j+3}, \\
& x \in \lambda_{i}, i: N-1,
\end{aligned}
$$

where $\chi \in I$. Applying the results of Blank [30], we may evaluate the fractional differential operator of order $q$ for the the collocation solution (23) at $x=x_{i}+c_{i} h$ as follows:

$$
\begin{aligned}
& D^{(\alpha)}\left(u_{i}\left(x_{i}+c_{j} h\right)\right)=\frac{N^{\alpha}}{\Gamma(1-\alpha)}\left(\sum_{k=0}^{i} \sum_{r=0}^{3} w_{j, r}^{(i-k, \alpha)} \mu_{r}^{(k)}\right. \\
& \left.\quad+\sum_{k=0}^{i} \sum_{s=0}^{m} w_{j, s+3}^{(i-k, \alpha)} \beta_{s}^{(k)}\right),
\end{aligned}
$$

where

$$
w_{i, k}^{(j, \alpha)}= \begin{cases}\left(j+c_{i}\right)^{-\alpha}-\delta_{j, 0}^{*}\left(j+c_{i}-1\right)^{-\alpha}, & k=0 \\ \left(j+c_{i}\right)^{-\alpha+k} \prod_{l=1}^{k} \frac{l}{l-\alpha}-\delta_{j, 0}^{*}\left(j+c_{i}-1\right)^{\nu-\alpha} \prod_{l=1}^{\nu} \frac{k-\nu+l}{l-\alpha}, & k \geq 1\end{cases}
$$

and $\delta_{j, 0}^{*}=0$ if $j=0$, and 1 otherwise. Consequently, (21) for each $X_{i}(i=0,1, \ldots, N-1)$ can be expressed in the form

$$
\begin{aligned}
& \frac{N^{\alpha}}{\Gamma(1-\alpha)}\left(\sum_{k=0}^{i} \sum_{r=0}^{3} w_{l, r}^{(i-k, \alpha)} \mu_{r}^{(k)}+\sum_{k=0}^{i} \sum_{s=0}^{m} w_{l, s+3}^{(i-k, \alpha)} \beta_{s}^{(k)}\right) \\
& \quad+\int_{0}^{x_{i, l}} K\left(x_{i, l}, t\right) f\left(t, \sum_{q=0}^{3} \mu_{q}^{(i)} c_{l}^{q}+\sum_{j=1}^{m} \beta_{j}^{(i)} c_{l}^{j+3}\right) d t \\
& \quad+h\left(x_{i, l}\right)=0,
\end{aligned}
$$

for $l=1,2, \ldots, N$. Applying Simpson's rule to approximate the integral in (26), one obtains

$$
\begin{aligned}
& \frac{N^{\alpha}}{\Gamma(1-\alpha)}\left(\sum_{k=0}^{i} \sum_{r=0}^{3} w_{l, r}^{(i-k, \alpha)} \mu_{r}^{(k)}+\sum_{k=0}^{i} \sum_{s=1}^{m} w_{l, s+3}^{(i-k, \alpha)} \beta_{s}^{(k)}\right) \\
& +\frac{x_{i, l}}{3} K\left(x_{i, l}, 0\right) f\left(0, \sum_{q=0}^{3} \mu_{q}^{(i)} c_{l}^{q}+\sum_{j=1}^{m} \beta_{j}^{(i)} c_{l}^{j+3}\right) \\
& +\frac{4 x_{i, l}}{3} K\left(x_{i, l}, \frac{x_{i, l}}{2}\right) f\left(\frac{x_{i, l}}{2}, \sum_{q=0}^{3} \mu_{q}^{(i)} c_{l}^{q}+\sum_{j=1}^{m} \beta_{j}^{(i)} c_{l}^{j+3}\right) \\
& +\frac{x_{i, l}}{3} K\left(x_{i, l}, x_{i, l}\right) f\left(x_{i, l}, \sum_{q=0}^{3} \mu_{q}^{(i)} c_{l}^{q}+\sum_{j=1}^{m} \beta_{j}^{(i)} c_{l}^{j+3}\right) \\
& +h\left(x_{i, l}\right)=0 .
\end{aligned}
$$

Obviously, (27) can be written in the following matrix form:

$$
g_{i}\left(M^{(i)}, B^{(i)}\right)=R^{(i)}, \quad i=0,1, \ldots, N-1,
$$

where

$$
\begin{aligned}
& g_{i l}\left(M^{(i)}, B^{(i)}\right)=\frac{N^{\alpha}}{\Gamma(1-\alpha)}\left(\sum_{k=0}^{i} \sum_{r=0}^{3} w_{l, r}^{(i-k, \alpha)} \mu_{r}^{(k)}\right. \\
& \left.+\sum_{k=0}^{i} \sum_{s=1}^{m} w_{l, s+3}^{(i-k, \alpha)} \beta_{s}^{(k)}\right)+\frac{x_{i, l}}{3} K\left(x_{i, l}, 0\right) \\
& +f\left(0, \sum_{q=0}^{3} \mu_{q}^{(i)} c_{l}^{q}+\sum_{j=1}^{m} \beta_{j}^{(i)} c_{l}^{j+3}\right) \\
& +\frac{4 x_{i, l}}{3} K\left(x_{i, l}, \frac{x_{i, l}}{2}\right) f\left(\frac{x_{i, l}}{2}, \sum_{q=0}^{3} \mu_{q}^{(i)} c_{l}^{q}\right. \\
& \left.+\sum_{j=1}^{m} \beta_{j}^{(i)} c_{l}^{j+3}\right)+\frac{x_{i, l}}{3} K\left(x_{i, l}, x_{i, l}\right) f\left(x_{i, l}, \sum_{q=0}^{3} \mu_{q}^{(i)} c_{l}^{q}\right. \\
& \left.+\sum_{j=1}^{m} \beta_{j}^{(i)} c_{l}^{j+3}\right), \quad l=1,2, \ldots, N,
\end{aligned}
$$

$M^{(i)}=\left[\mu_{0}^{(i)}, \ldots, \mu_{3}^{(i)}\right]^{t}, B^{(i)}=\left[\beta_{1}^{(i)}, \ldots, \beta_{m}^{(i)}\right]^{t}$, and $R^{(i)}=$ $\left[-h\left(x_{i, 1}\right), \ldots,-h\left(x_{i, N}\right)\right]^{t}$. Here $[\cdot]^{t}$ means the transpose of the vector. Based on the given definition of the approximate spline $u$, it can be easily verified that

$$
M^{(0)}=\left[\begin{array}{l}
\mu_{0}^{(0)} \\
\mu_{1}^{(0)} \\
\mu_{2}^{(0)} \\
\mu_{3}^{(0)}
\end{array}\right]=\left[\begin{array}{l}
a_{0} \\
a_{1} \\
a_{2} \\
a_{3}
\end{array}\right] .
$$


4.2. Multiple Shooting Method. For simplicity, let us rewrite problem (1)-(2b) in the following form:

$$
\mathscr{L} y:=D^{\alpha} y(x)+\int_{0}^{x} K(x, t) f(t, y) d t=-h(x),
$$

subject to

$$
\begin{gathered}
y(0)=a_{0}, \\
y^{\prime}(0)=a_{1}, \\
y(1)=b_{0}, \\
y^{\prime}(1)=b_{1} .
\end{gathered}
$$

The solution of problem (31)-(32) can be determined by solving the problem on the subintervals $\left[x_{i}, x_{i+1}\right]$, for $i=$ $0,1, \ldots, N-1$. In order to apply the shooting method, we introduce the following set of initial value problems:

$$
\begin{array}{r}
\mathscr{L}\left[y_{i}\right]=D^{\alpha} y_{i}(x)+\int_{0}^{x} K(x, t) f\left(t, y_{i}\right) d t=-h(x), \\
x_{i} \leq x \leq x_{i+1}
\end{array}
$$

subject to

$$
\begin{gathered}
y_{i}\left(x_{i}\right)=a_{4 i}, \\
y_{i}^{\prime}\left(x_{i}\right)=a_{4 i+1}, \\
y_{i}^{\prime \prime}\left(x_{i}\right)=a_{4 i+2}, \\
y_{i}^{\prime \prime \prime}\left(x_{i}\right)=a_{4 i+3},
\end{gathered}
$$

where $a_{2}, a_{3}, \ldots, a_{4 N-1}$ are unknown real parameters. The solution of problem (33)-(34) will be obtained by the method described in the previous subsection, where the parameters $a_{2}, a_{3}, \ldots, a_{4 N-1}$ are determined by solving the following algebraic system

$$
\begin{gathered}
y_{0}^{(n)}\left(x_{1}, a_{2}: a_{3}\right)=y_{1}^{(n)}\left(x_{1}, a_{4}: a_{7}\right), \\
\vdots \\
y_{i-1}^{(n)}\left(x_{i}, a_{4 i-4}: a_{4 i-1}\right)=y_{i}^{(n)}\left(x_{i}, a_{4 i}: a_{4 i+3}\right), \\
\vdots \\
y_{N-1}\left(1, a_{4 N-4}: a_{4 N-1}\right)=b_{0}, \\
y_{N-1}^{\prime}\left(1, a_{4 N-4}: a_{4 N-1}\right)=b_{1},
\end{gathered}
$$

where $n=0: 3$ and $a_{l}: a_{m}$ represents $a_{l}, a_{l+1}, \ldots, a_{m}$. It is worth mentioning that we use shooting method of order five $(N=5)$ in our computations which are done using Matlab.

\section{Numerical Results}

Example 1. Consider the following nonlinear fourth order fractional integrodifferential equations:

$$
D^{\alpha} y(x)+\int_{0}^{x}(t+1) y^{2}(t) d t+h(x)=0
$$

$$
0<x<1
$$

subject to

$$
\begin{gathered}
y(0)=1, \\
y^{\prime}(0)=0, \\
y(1)=2, \\
y^{\prime}(1)=3,
\end{gathered}
$$

where $\alpha=3.7, h(x)=-x^{8} / 8-x^{7} / 7-2 x^{5} / 5-x^{4} / 2-x^{2} / 2-x$, and the exact solution is $y(x)=x^{3}+1$. Divide the domain $[0,1]$ into five subinterval such that

$$
\bigcup_{i=0}^{4}\left[x_{i}, x_{i-1}\right] \quad \text { where } x_{i}=0.2 i, i=0,1, \ldots, 5 \text {, }
$$

and then apply the multiple shooting method of order five yields to solve the following five initial value problems:

$$
\begin{aligned}
\mathscr{L}\left[y_{0}\right] & =-h(x), \quad 0 \leq x \leq 0.2, \\
y_{0}(0,:) & =1, \\
y_{0}^{\prime}(0,:) & =3, \\
y_{0}^{\prime \prime}(0,:) & =a_{2}, \\
y_{0}^{\prime \prime \prime}(0,:) & =a_{3}, \\
\mathscr{L}\left[y_{1}\right] & =-h(x), \quad 0.2 \leq x \leq 0.4, \\
y_{1}(0.2,:) & =a_{4}, \\
y_{1}^{\prime}(0.2,:) & =a_{5}, \\
y_{1}^{\prime \prime}(0.2,:) & =a_{6}, \\
y_{1}^{\prime \prime \prime}(0.2,:) & =a_{7}, \\
\mathscr{L}\left[y_{2}\right] & =-h(x), \quad 0.4 \leq x \leq 0.6, \\
y_{2}(0.4,:) & =a_{8}, \\
y_{2}^{\prime}(0.4,:) & =a_{9}, \\
y_{2}^{\prime \prime}(0.4,:) & =a_{10}, \\
y_{2}^{\prime \prime \prime}(0.4,:) & =a_{11}, \\
\mathscr{L}\left[y_{3}\right] & =-h(x), \quad 0.6 \leq x \leq 0.8,
\end{aligned}
$$




$$
\begin{aligned}
y_{3}(0.6,:) & =a_{12}, \\
y_{3}^{\prime}(0.6,:) & =a_{13}, \\
y_{3}^{\prime \prime}(0.6,:) & =a_{14}, \\
y_{4}^{\prime \prime \prime}(0.6,:) & =a_{15}, \\
\mathscr{L}\left[y_{4}\right] & =-h(x), \quad 0.8 \leq x \leq 1.0, \\
y_{4}(0.8,:) & =a_{16}, \\
y_{4}^{\prime}(0.8,:) & =a_{17}, \\
y_{4}^{\prime \prime}(0.8,:) & =a_{18}, \\
y_{4}^{\prime \prime \prime}(0.8,:) & =a_{19},
\end{aligned}
$$

where

$$
\mathscr{L}[y]=D^{\alpha} y(x)+\int_{0}^{x}(t+1) y^{2}(t) d t
$$

Here $y_{0}(x,:)=y_{0}\left(x, a_{2}, a_{3}\right), y_{1}(x,:)=y_{1}\left(x, a_{4}: a_{7}\right)$, $y_{2}(x,:)=y_{2}\left(x, a_{8}: a_{11}\right), y_{3}(x,:)=y_{3}\left(x, a_{12}: a_{15}\right)$, and $y_{4}(x,:)=y_{4}\left(x, a_{16}: a_{19}\right)$. The above initial value problems (39) are solved using the collocation method with the following collocation points:

$$
\begin{aligned}
& c_{1}=\frac{1+\cos (\pi / 8)}{2}, \\
& c_{2}=\frac{1+\cos (3 \pi / 8)}{2}, \\
& c_{3}=\frac{1+\cos (5 \pi / 8)}{2}, \\
& c_{4}=\frac{1+\cos (7 \pi / 8)}{2} .
\end{aligned}
$$

Notice that these collocations points are generated from the roots of the Chebyshev polynomials of degree four. To find the parameters $a_{i}, i=2: 19$ we solve the following algebraic system:

$$
\begin{aligned}
y_{0}^{(n)}\left(0.2, a_{2}: a_{3}\right) & =y_{1}^{(n)}\left(0.2, a_{4}: a_{7}\right), \\
y_{1}^{(n)}\left(0.4, a_{4}: a_{7}\right) & =y_{2}^{(n)}\left(0.4, a_{8}: a_{11}\right), \\
y_{2}^{(n)}\left(0.6, a_{8}: a_{11}\right) & =y_{3}^{(n)}\left(0.6, a_{12}: a_{15}\right), \\
y_{3}^{(n)}\left(0.8, a_{12}: a_{15}\right) & =y_{4}^{(n)}\left(0.8, a_{16}: a_{19}\right), \\
y_{4}\left(1, a_{16}: a_{19}\right) & =2, \\
y_{4}^{\prime}\left(1, a_{16}: a_{19}\right) & =3,
\end{aligned}
$$

for $n=0,1,2,3$, using Matlab software. Hence, we obtain

$$
\begin{aligned}
& a_{2}=5.999999998, \\
& a_{3}=6.000000001, \\
& a_{4}=1.008000002, \\
& a_{5}=0.120000003, \\
& a_{6}=1.199999998, \\
& a_{7}=6.000000001, \\
& a_{8}=1.063999996, \\
& a_{9}=0.48000005, \\
& a_{10}=2.40000002, \\
& a_{11}=6.000000002, \\
& a_{12}=1.21600007, \\
& a_{13}=1.08000008, \\
& a_{14}=3.599999995, \\
& a_{15}=5.999999994, \\
& a_{16}=1.51199989, \\
& a_{17}=1.919999990, \\
& a_{18}=4.800000012, \\
& a_{19}=5.999999986
\end{aligned}
$$

The graph of the exact and approximate solutions and the graph of the error function are, respectively, given in Figures 1 and 2. It is clearly seen that the two solutions are in excellent agreement. In addition, the computed $L_{2}$ error norm is given by

$$
\begin{aligned}
\|u(x)-y(x)\| & =\sum_{i=0}^{4} \int_{x_{i}}^{x_{i+1}}\left(u(x)-y_{i+1}(x)\right)^{2} d x \\
& =2.1 \times 10^{-14} .
\end{aligned}
$$

Example 2. Consider the following nonlinear fourth order fractional integrodifferential equations:

$$
\begin{array}{r}
D^{\alpha} y(x)+\int_{0}^{x} t^{2} y(t) d t-\left(\frac{x^{3}}{\alpha+3}+1\right) E_{\alpha, 1}\left(x^{\alpha}\right)=0 \\
0<x<1
\end{array}
$$

subject to

$$
\begin{aligned}
y(0) & =1, \\
y^{\prime}(0) & =\frac{1}{\alpha \Gamma(\alpha)}, \\
y(1) & =E_{\alpha, 1}(1), \\
y^{\prime}(1) & =\frac{E_{\alpha, \alpha}(1)}{\alpha},
\end{aligned}
$$




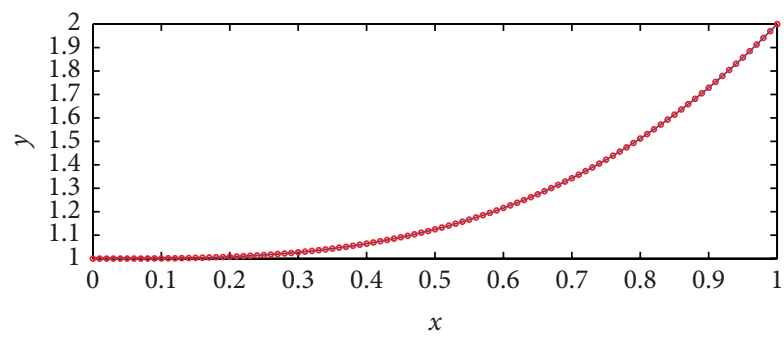

Exact solution

- Approximate solution

Figure 1: The exact and approximate solutions for Example 1.

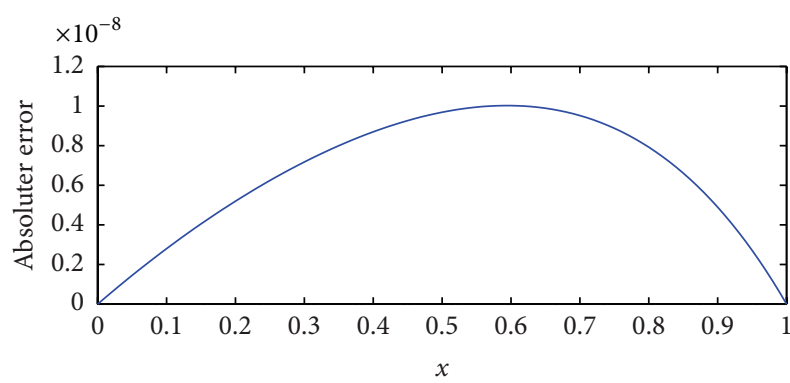

Figure 2: Computed absolute error between the present numerical solution and the exact one for Example 1.

where $\alpha=3.5$ and the exact solution is $y(x)=E_{\alpha, 1}\left(x^{\alpha}\right)$. Here $E_{\alpha, \beta}(z)=\sum_{n=0}^{\infty}\left(z^{n} / \Gamma(\alpha n+\beta)\right)$ represents the Mittag-Leffler function. Using the multiple shooting method of order five and then following the same steps in the previous example, the parameters $a_{i}, i=2,3, \ldots, 19$ are found to be

$$
\begin{aligned}
& a_{2}=0.000396825, \\
& a_{3}=0.0000000504, \\
& a_{4}=1.0172022865, \\
& a_{5}=0.086051128, \\
& a_{6}=0.0003969266, \\
& a_{7}=0.0000000503, \\
& a_{8}=1.034420446, \\
& a_{9}=1.49160490, \\
& a_{10}=0.000397028, \\
& a_{11}=0.0000000504, \\
& a_{12}=1.051654498, \\
& a_{13}=0.086209945, \\
& a_{14}=0.000397123,
\end{aligned}
$$

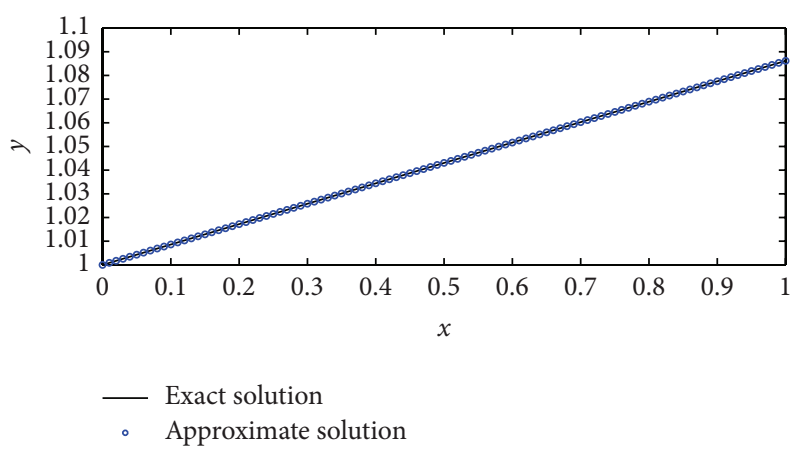

FIgUre 3: The exact and approximate solutions for Example 2.

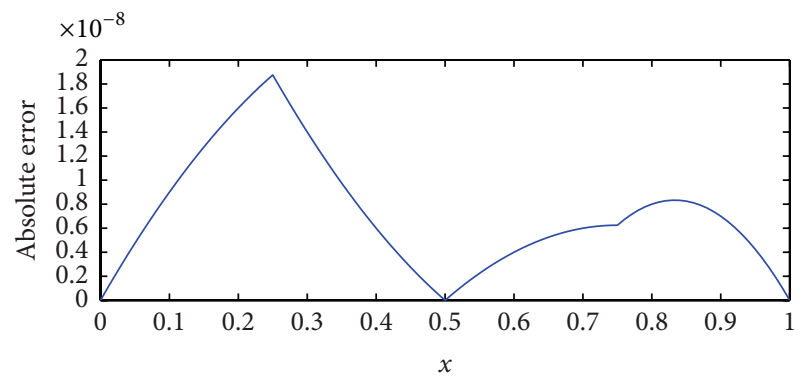

FIgURE 4: Computed absolute error between the present numerical solution and the exact one for Example 2.

$$
\begin{aligned}
& a_{15}=0.0000000505, \\
& a_{16}=1.068904427, \\
& a_{17}=0.086289345, \\
& a_{18}=0.000397224, \\
& a_{19}=0.0000000504 .
\end{aligned}
$$

The exact solution is graphed along with the graph of the approximate solution for $\alpha=3.5$ in Figure 3. Moreover, the computed absolute error between the numerical and exact solutions is given in Figure 4 which shows a satisfactory agreement. The computed $L_{2}$ error norm is given by

$$
\begin{aligned}
\|u(x)-y(x)\| & =\sum_{i=0}^{4} \int_{x_{i}}^{x_{i+1}}\left(u(x)-y_{i+1}(x)\right)^{2} d x \\
& =1.87 \times 10^{-15} .
\end{aligned}
$$

Example 3. Consider the following nonlinear fourth order fractional integrodifferential equations

$$
D^{\alpha} y(x)+\int_{0}^{x} y^{2}(t) d t+H(x)=0, \quad 0<x<1
$$


subject to

$$
\begin{gathered}
y(0)=0, \\
y^{\prime}(0)=1, \\
y(1)=0.84, \\
y^{\prime}(1)=0.54,
\end{gathered}
$$

where $\alpha=3.25$ and $H(x)=0.005-0.13 x-x^{2}$. By implementing the present algorithm, the parameters $a_{i}, i=2,3, \ldots, 19$ are found to be

$$
\begin{aligned}
& a_{2}=0, \\
& a_{3}=-1, \\
& a_{4}=0.198669, \\
& a_{5}=0.980067, \\
& a_{6}=-0.198669, \\
& a_{7}=-0.980067, \\
& a_{8}=0.389418, \\
& a_{9}=0.921061, \\
& a_{10}=-0.389418, \\
& a_{11}=-0.921061, \\
& a_{12}=0.564642, \\
& a_{13}=0.825336, \\
& a_{14}=-0.564642 \\
& a_{15}=-0.825336, \\
& a_{16}=0.717356, \\
& a_{17}=0.696707 \\
& a_{18}=-0.717356, \\
& a_{19}=-0.696707 .
\end{aligned}
$$

Figure 5 shows the graph of the approximate solution. Since the actual solution for this problem is unknown, we may measure the error of the approximation by using the residual function, $R(x)$, defined by

$$
R(x):=D^{\alpha} u(x)+\int_{0}^{x} u^{2}(t) d t+H(x) .
$$

The graph of the residual function, $R(x)$, is displayed in Figure 6. It is clearly evident from Figure 6 that $\operatorname{Max}\{|R(x)|$ : $0 \leq x \leq 1\}=0.0056$.

\section{Conclusion and Future Work}

In this paper, we have solved special class of higher-order nonlinear integrodifferential equations of order 4 subject to

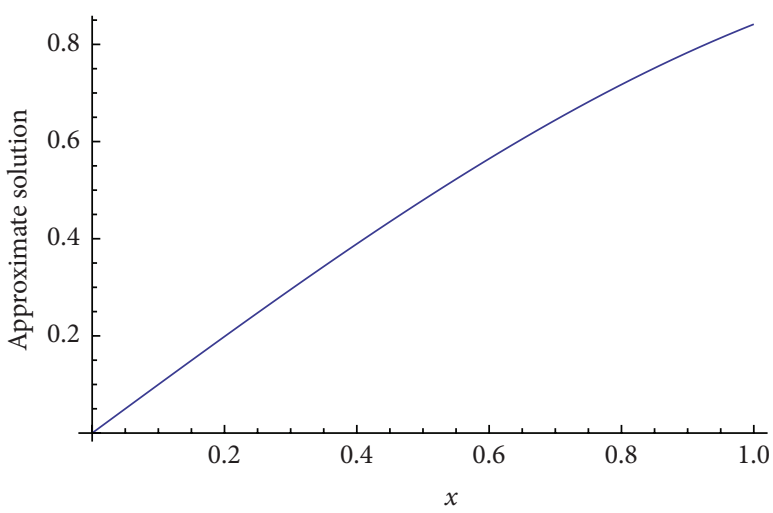

Figure 5: The exact and approximate solutions for Example 3.

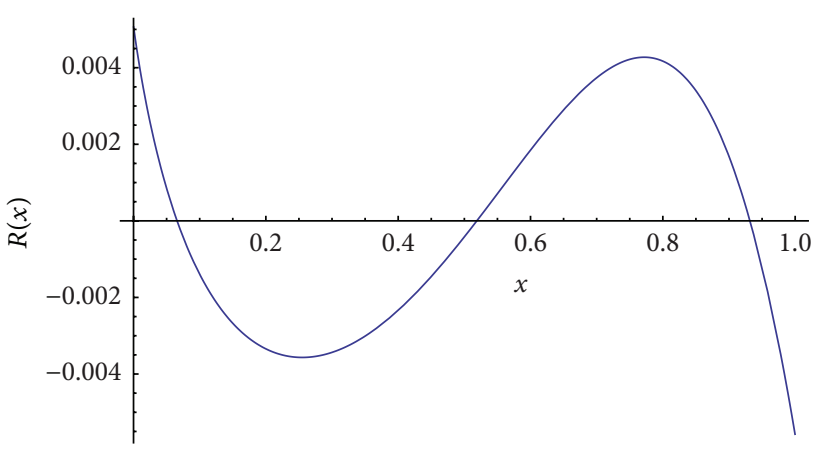

Figure 6: Computed residual, $R(x)$, for Example 3.

boundary conditions. The method of solution is based on conjugate collocation and spline technique with shooting method. The numerical results for given examples demonstrate the efficiency and accuracy of the present method. It should be noted that applying Theorem 5 causes two difficulties. Firstly, the Lipschitz constant for $f(x, y)$ with respect to $y$ is not easy to find and, secondly, the necessary condition $(0<L M / \alpha \Gamma(\alpha)<1)$ does not satisfy several cases of the problem (1)-(2b). Therefore, a further discussion on weaker necessary conditions should be followed in the future work to cover wider range of problem (1)-(2b).

\section{Conflict of Interests}

The authors declare that there is no conflict of interests regarding the publication of this paper.

\section{Acknowledgments}

The authors would like to express their appreciation for the valuable comments of the reviewers. The authors also would like to express their sincere appreciation to the United Arab Emirates University Research Affairs for the financial support of Grant no. COS/IRG-16/14. 


\section{References}

[1] R. P. Agarwal, S. K. Ntouyas, B. Ahmad, and M. S. Alhothuali, "Existence of solutions for integro-differential equations of fractional order with nonlocal three-point fractional boundary conditions," Advances in Difference Equations, vol. 2013, article 128, 2013.

[2] I. Podlubny, Fractional Differential Equations, Academic Press, San Diego, Calif, USA, 1999.

[3] F. Mainardi, "Fractional calculus: Some basic problems in continuum and statistical mechanics," in Fractals and Fractional Calculus in Continuum Mechanics, A. Carpinteri and F. Mainardi, Eds., pp. 223-276, Springer, Vienna, Austria, 1997.

[4] A. A. Kilbas, H. M. Srivastava, and J. J. Trujillo, Theory and Applications of Fractional Differential Equations, vol. 204 of North-Holland Mathematics Studies, Elsevier, Amsterdam, The Netherlands, 2006.

[5] B. Ahmad and B. S. Alghamdi, "Approximation of solutions of the nonlinear Duffing equation involving both integral and non-integral forcing terms with separated boundary conditions," Computer Physics Communications, vol. 179, no. 6, pp. 409-416, 2008.

[6] Y.-K. Chang and J. J. Nieto, "Existence of solutions for impulsive neutral integro-differential inclusions with nonlocal initial conditions via fractional operators," Numerical Functional Analysis and Optimization, vol. 30, no. 3-4, pp. 227-244, 2009.

[7] Z. Luo and J. J. Nieto, "New results for the periodic boundary value problem for impulsive integro-differential equations," Nonlinear Analysis, Theory, Methods \& Applications, vol. 70, no. 6, pp. 2248-2260, 2009.

[8] S. Mesloub, "On a mixed nonlinear one point boundary value problem for an integrodifferential equation," Boundary Value Problems, vol. 2008, Article ID 814947, 8 pages, 2008.

[9] R. P. Agarwal, B. De Andrade, and G. Siracusa, "On fractional integro-differential equations with state-dependent delay," Computers and Mathematics with Applications, vol. 62, no. 3, pp. 1143-1149, 2011.

[10] B. Ahmad and J. J. Nieto, "Existence results for nonlinear boundary value problems of fractional integrodifferential equations with integral boundary conditions," Boundary Value Problems, vol. 2009, Article ID 708576, 2009.

[11] B. Ahmad and S. Sivasundaram, "On four-point nonlocal boundary value problems of nonlinear integro-differential equations of fractional order," Applied Mathematics and Computation, vol. 217, no. 2, pp. 480-487, 2010.

[12] M. Alipour and D. Baleanu, "Approximate analytical solution for nonlinear system of fractional differential equations by BPs operational matrices," Advances in Mathematical Physics, vol. 2013, Article ID 954015, 9 pages, 2013.

[13] Q. M. Al-Mdallal, "On the numerical solution of fractional Sturm-Liouville problems," International Journal of Computer Mathematics, vol. 87, no. 12, pp. 2837-2845, 2010.

[14] Q. M. Al-Mdallal and M. I. Syam, "An efficient method for solving non-linear singularly perturbed two points boundaryvalue problems of fractional order," Communications in Nonlinear Science and Numerical Simulation, vol. 17, no. 6, pp. 22992308, 2012.

[15] Q. M. Al-Mdallal, M. I. Syam, and M. N. Anwar, "A collocationshooting method for solving fractional boundary value problems," Communications in Non-Linear Science and Numerical Simulation, vol. 15, no. 12, pp. 3814-3822, 2010.
[16] J. Cao, Q. Yang, and Z. Huang, "Optimal mild solutions and weighted pseudo-almost periodic classical solutions of fractional integro-differential equations," Nonlinear Analysis, Theory, Methods and Applications, vol. 74, no. 1, pp. 224-234, 2011.

[17] M. H. M. Rashid and Y. El-Qaderi, "Semilinear fractional integro-differential equations with compact semigroup," Nonlinear Analysis: Theory, Methods \& Applications, vol. 71, no. 12, pp. 6276-6282, 2009.

[18] R. C. Mittal and R. Nigam, "Solution of fractional integrodifferential equations by Adomian decomposition method," International Journal of Applied Mathematics and Mechanics, vol. 4, no. 2, pp. 87-94, 2008.

[19] S. Momani and M. Aslam Noor, "Numerical methods for fourth-order fractional integro-differential equations," Applied Mathematics and Computation, vol. 182, no. 1, pp. 754-760, 2006.

[20] E. A. Rawashdeh, "Numerical solution of fractional integrodifferential equations by collocation method," Applied Mathematics and Computation, vol. 176, no. 1, pp. 1-6, 2006.

[21] J. Zhao, J. Xiao, and N. J. Ford, "Collocation methods for fractional integro-differential equations with weakly singular kernels," Numerical Algorithms, vol. 65, no. 4, pp. 723-743, 2014.

[22] Y. Nawaz, "Variational iteration method and homotopy perturbation method for fourth-order fractional integro-differential equations," Computers and Mathematics with Applications, vol. 61, no. 8, pp. 2330-2341, 2011.

[23] K. Sayevand, "Analytical treatment of Volterra integrodifferential equations of fractional order," Applied Mathematical Modelling, vol. 39, no. 15, pp. 4330-4336, 2015.

[24] A. Arikoglu and I. Ozkol, "Solution of fractional integrodifferential equations by using fractional differential transform method," Chaos, Solitons and Fractals, vol. 40, no. 2, pp. 521-529, 2009.

[25] D. Nazari and S. Shahmorad, "Application of the fractional differential transform method to fractional-order integro-differential equations with nonlocal boundary conditions," Journal of Computational and Applied Mathematics, vol. 234, no. 3, pp. 883-891, 2010.

[26] H. Saeedi and M. M. Moghadam, "Numerical solution of nonlinear Volterra integro-differential equations of arbitrary order by CAS wavelets," Communications in Nonlinear Science and Numerical Simulation, vol. 16, no. 3, pp. 1216-1226, 2011.

[27] P. Mokhtary, "Discrete Galerkin method for fractional integrodifferential equations," Acta Mathematica Scientia, http://arxiv .org/abs/1501.01111.

[28] J. Biazar and H. Ebrahimi, "Chebyshev wavelets approach for nonlinear systems of Volterra integral equations," Computers and Mathematics with Applications, vol. 63, no. 3, pp. 608-616, 2012.

[29] L. Huang, X.-F. Li, Y. Zhao, and X.-Y. Duan, "Approximate solution of fractional integro-differential equations by Taylor expansion method," Computers and Mathematics with Applications, vol. 62, no. 3, pp. 1127-1134, 2011.

[30] L. Blank, "Numerical treatment of differential equations of fractional order," Numerical Analysis Report 287, Manchester Center for Numerical Computational Mathematics, 1996. 


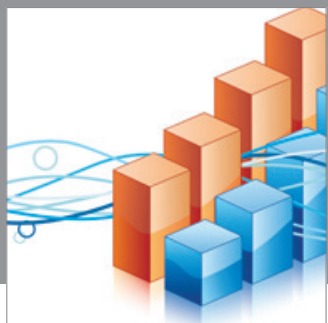

Advances in

Operations Research

mansans

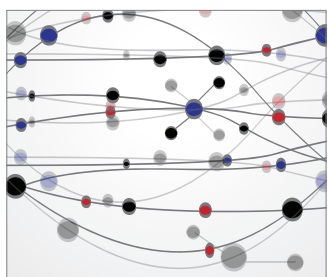

The Scientific World Journal
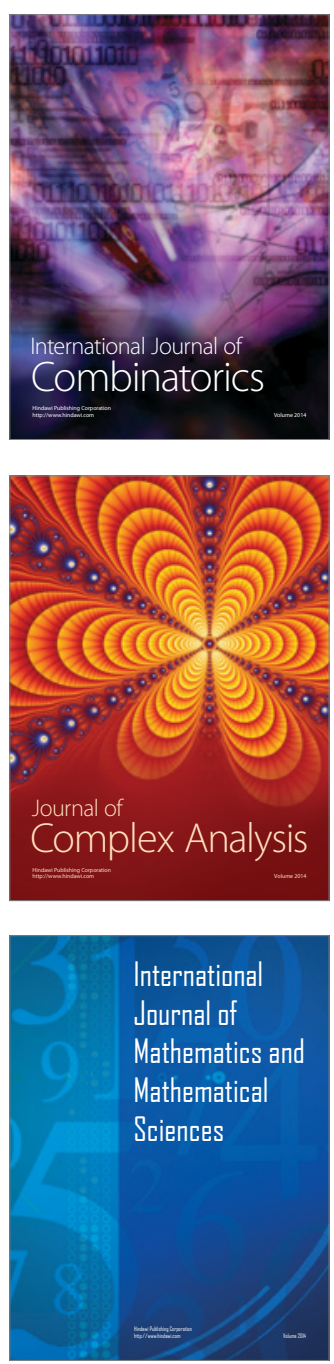
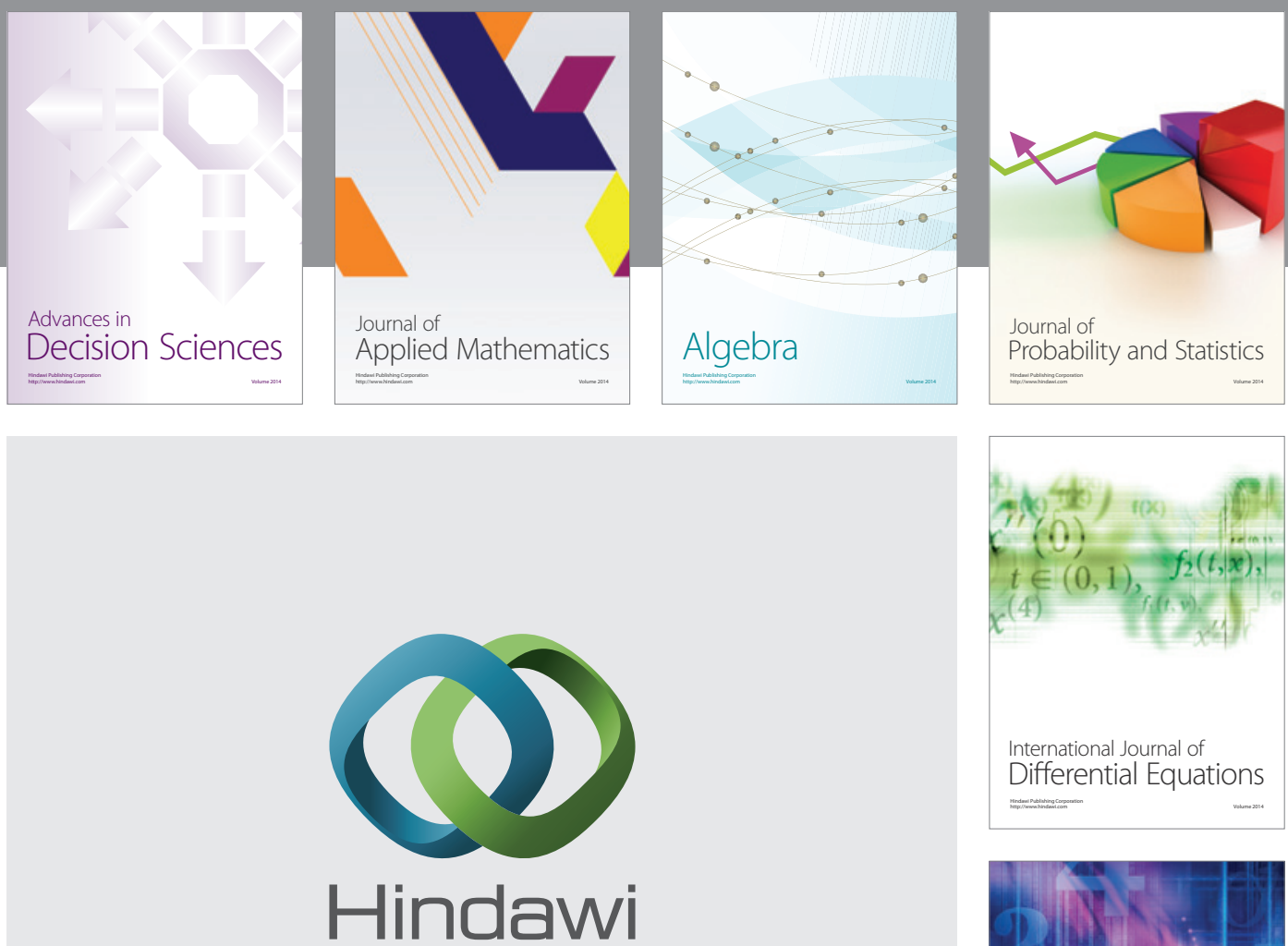

Submit your manuscripts at http://www.hindawi.com
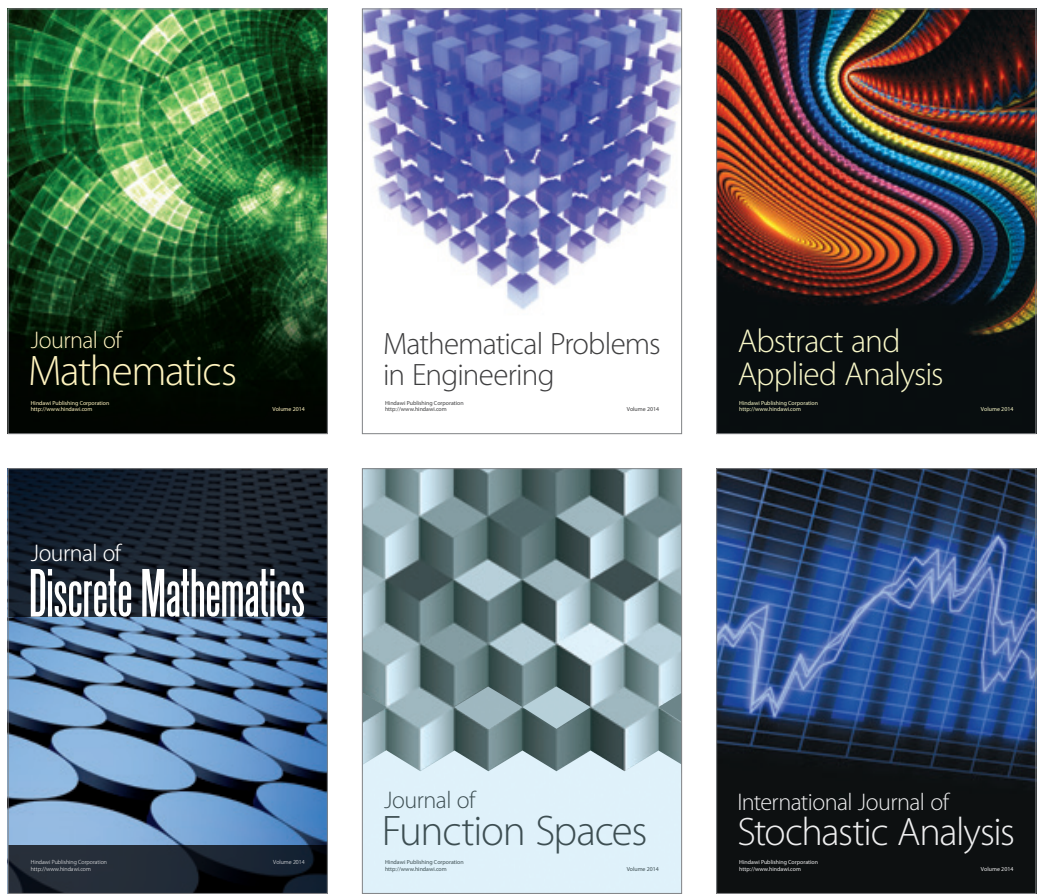

Journal of

Function Spaces

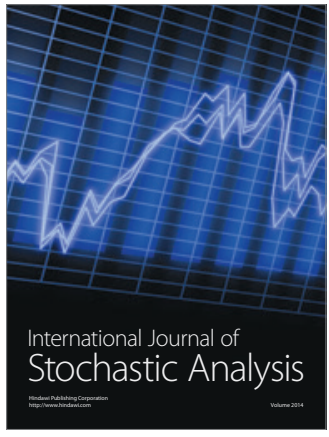

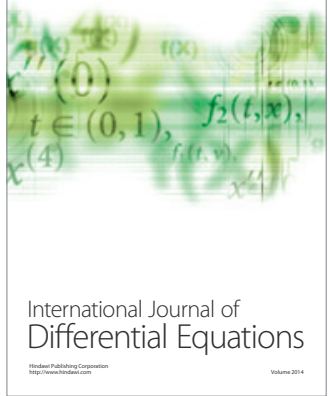
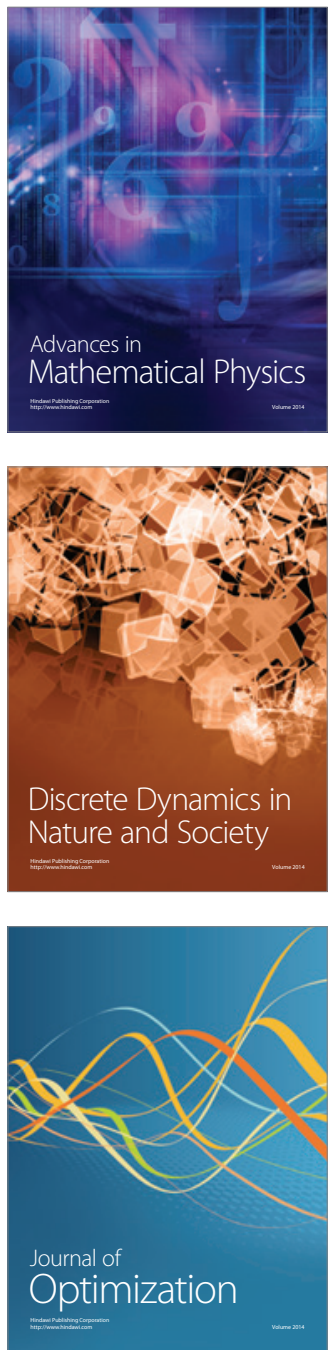